\title{
Adaptive parameter estimation for an energy model of belt conveyor with
}

\section{DC motor}

\author{
Yanjun Shen, Xiaohua Xia
}

\begin{abstract}
In practice, design parameters of a belt conveyor are likely drifting away from their design values by maintenance, readjustment, retrofit, abrasion and circumstance change. For the purpose of energy optimization, these parameters should be estimated through experiments. In this paper, a new energy model of a DC motor driven belt conveyor is presented. Then, based on an adaptive observer, a parameter estimation algorithm is derived. In addition, under a persistent excitation condition, the convergence of the parameters to the desired values can also be concluded. Compared with the existing methods, our methods can be implemented by measuring only the feed rate of the belt conveyor and the angular velocity of the rotor of the DC motor.
\end{abstract}

Key Words: parameters estimation, energy model, belt conveyor, observer, DC motor

\section{Introduction}

Y. Shen is with the College of Electrical, Engineering and New Energy, China Three Gorges University, Yichang, Hubei, 443002, China.

X. Xia is with Department of Electrical, Electronic and Computer Engineering, University of Pretoria, Pretoria 0002, South Africa

This work was supported by the National Science Foundation of China (No. 61074091, 61174216, 61273183), the National Science Foundation of Hubei Province (2011CDB187), the Scientific Innovation Team Project of Hubei Provincial Department of Education (T201103).
Belt conveyors have high transfer capacity and long transfer distance. They are widely used to transfer bulk material in mining, metallurgical and coal industry. According to the report in [1], about $10 \%$ of the total maximum power demand in South Africa is to handle materials, where up to $40 \%$ energy cost is borne by the operational cost of the belt conveyor systems [2]. Therefore, it has great significance to improve energy efficiency of belt conveyors by reducing the energy consumption of material handling. 
There are four levels to improve energy efficiency of a belt conveyor: performance, operation, equipment, and technology [3]. It is easy to achieve higher energy efficiency by introducing highly efficient equipment [4, $5,6,7,8]$. However, extra investment is needed to retrofit or replace the equipment. At the operation level, many methods are proposed to improve energy efficiency for the belt conveyors $[9,10,11,12,13$, $14,15,16,17,18]$. For example, the authors in [17] proposed an optimal switching control and a variable speed drive based optimal control to reduce the energy consumption of belt conveyors. In [18], an analytical energy model is proposed. It has four coefficients which can be estimated through the algorithms such as least square (LSQ) [19] and recursive least square (RLSQ) [20]. After obtaining the energy model, an optimization is also done at operational level with two performance indicators, energy cost and energy consumption. However, in order to estimate these four coefficients, power of motor $P_{M}$, feed rate $T$ and belt speed $V$ should be measured. Recently, in order to estimate unknown states and unknown constant parameters, adaptive observers have made great progress [21, 22, 23, 24, 25, 26, 27, 28, 29, 30].

In this paper, a new energy model of belt conveyor with DC motor is introduced. Then, an adaptive observer is designed for the model. In order to identify the four coefficients of the energy model, the feed rate $T$ of the belt conveyor and the angular velocity $w_{m}$ of the rotor of the DC motor should be measured online. Then, based on the adaptive observer, a parameter estimation algorithm is derived. In addition, under a persistent excitation condition, the convergence of the parameters to the desired values can also be concluded. Simulation results show the validity of our methods.
This paper is organized as follows. The analytical energy model of belt conveyors in [18], the model of a DC motor, and the adaptive observers design are reviewed in Section II, respectively. In Section III, we present a new energy model of belt conveyors with DC motor, an adaptive observer for this model, and a parameter estimation algorithm. In Section IV, an example is given to show the validity of our new methods. Section V presents the conclusion.

\section{Preliminaries}

\subsection{An analytical energy model of belt conveyors}

A typical belt conveyor is shown in Fig. 1. As in [18], an analytical energy model of the belt conveyor is given as follows

$$
P_{T}-\frac{V^{2} T}{3.6}=\bar{\theta}_{1} T^{2} V+\bar{\theta}_{2} V+\bar{\theta}_{3} \frac{T^{2}}{V}+\bar{\theta}_{4} T
$$

where $P_{T}$ is the mechanical power, $V$ denotes the the belt speed $(\mathrm{m} / \mathrm{s}), T$ is the feed rate $(\mathrm{t} / \mathrm{h}), \bar{\theta}_{1}, \bar{\theta}_{2}, \bar{\theta}_{3}$ and $\bar{\theta}_{4}$ are four parameters. In practice, there four parameters often drift away by maintenance, readjustment, retrofit, abrasion and circumstance change. For the purpose of energy optimization, these four parameters are estimated by both an off-line and an on-line parameter estimation schemes based on $P_{T}, V$ and $T$ measured on-line and off-line, respectively in [18].

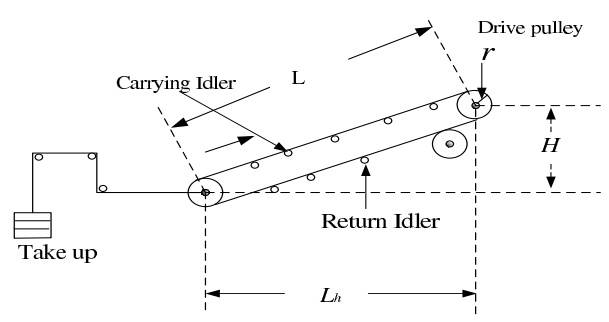

Fig. 1. Typical profile of belt conveyors. 


\subsection{A DC motor}

The dynamics of a DC motor are given by [31]

$$
\left\{\begin{aligned}
J_{m} \frac{d \omega_{m}}{d t}+b_{m} \omega_{m} & =k_{p} i_{f} i_{a}-T_{L} \\
L_{f} \frac{d i_{f}}{d t}+R_{f} i_{f} & =e_{f} \\
L_{a} \frac{d i_{a}}{d t}+R_{a} i_{a} & =e_{a}-k_{c} i_{f} \omega_{m}
\end{aligned}\right.
$$

where $J_{m}$ denotes the mass moment of inertia of the motor, $\omega_{m}$ is its angular velocity, $k_{p}$ is the torque constant, $b_{m}$ is the damping coefficient, $T_{L}$ is the presence of some external load, $L_{a}$ and $L_{f}$ are its inductances, $R_{a}$ and $R_{f}$ are its resistances, $i_{a}$ and $i_{f}$ are its currents, $k_{c}$ is proportional constant to the flux and the angular velocity of the motor, $e_{a}$ and $e_{f}$ are two separate potentials are used to power the armature and filed, respectively. The corresponding circuit is shown in Fig. 2.
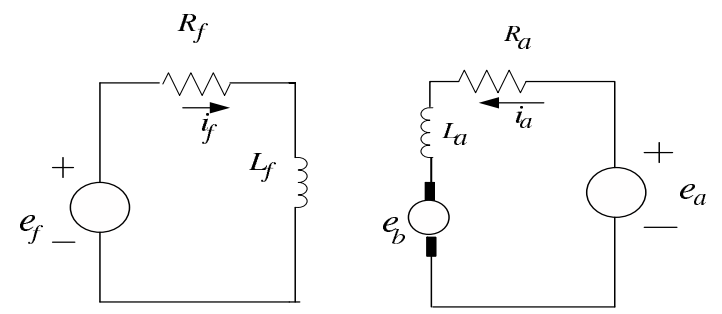

Fig. 2. Circuit diagram of separately excited DC motor.

\subsection{Adaptive observers}

Adaptive observers can be used to estimate unknown parameters. Now, let us review the adaptive observers design. Consider the following system in adaptive observer form [26]

$$
\left\{\begin{aligned}
\dot{z} & =\bar{A}_{0} z+\gamma(y, u)+\bar{b} \beta^{T}(y, u, t) \theta \\
y & =\bar{C}_{0} z
\end{aligned}\right.
$$

where $z \in \mathcal{R}^{n}, \quad y \in \mathcal{R}, \quad u \in \mathcal{R}^{m}, \quad \theta \in \mathcal{R}^{p}, \quad \gamma(y, u)$ a smooth function mapping $\mathcal{R} \times \mathcal{R}^{m} \rightarrow \mathcal{R}^{n}, \bar{A}_{0}=$ $\left[\begin{array}{cccc}0 & 1 & \cdots & 0 \\ \vdots & \vdots & \ddots & \vdots \\ 0 & 0 & \cdots & 1 \\ 0 & 0 & \cdots & 0\end{array}\right], \bar{b}=\left[\bar{b}_{1}, \cdots, \bar{b}_{n}\right]^{T} \in \mathcal{R}^{n}$ is given such that the polynomial $\bar{b}_{1} s^{n-1}+\cdots+\bar{b}_{n}$ is Hurwitz and $\bar{b}_{1}>0, \quad \bar{C}_{0}=\left[\begin{array}{lll}1 & 0 & \cdots\end{array}\right], \quad \beta^{T}(y, u, t) \theta=$ $\sum_{i=1}^{p} \theta_{i} \beta_{i}(y, u, t), \beta_{i}(y, u, t)(i=1, \cdots, p)$ are continuous functions and uniformly bounded for every $(y, u)$ bounded. A global adaptive observer with parameter convergence is designed in [26] as follows:

$$
\left\{\begin{aligned}
\dot{\hat{z}} & =\bar{A}_{0} \hat{z}+\bar{K} \bar{C}_{0} e+\gamma(y, u)+\bar{b} \beta^{T}(y, u, t) \hat{\theta}, \\
\dot{\hat{\theta}} & =\Gamma \beta(y, u, t) \bar{C}_{0} e,
\end{aligned}\right.
$$

where $\Gamma$ is any symmetric positive definite matrix, $e=$ $z-\hat{z}, \bar{K}=\frac{1}{b_{1}}\left(\bar{A}_{0} \bar{b}+\lambda \bar{b}\right)$ with $\lambda$ an arbitrary positive real. Since $\left(\bar{A}_{0}, \bar{b}, \bar{C}_{0}\right)$ satisfies the strictly positive real condition, then, for any symmetric definite matrix $\bar{Q}$, there exist a symmetric positive definite matrix $\bar{P}$, a positive real $\bar{d}$ such that [26]

$$
\left(\bar{A}_{0}+\bar{K} \bar{C}_{0}\right)^{T} \bar{P}+\bar{P}\left(\bar{A}_{0}+\bar{K} \bar{C}_{0}\right)<-\bar{d} \bar{Q}, \quad \bar{P} \bar{b}=\bar{C}^{T} .
$$

\section{A new energy model}

In this section, we make the following assumption: the belt is non-slip; $T$ and $\omega_{m}$ are measured on-line. We shall also assume a constant potential $e_{f}$ and assume that the circuit is operating at steady state so that $e_{f}=$ $i_{f} R_{f}$, yielding a constant field current $i_{f}$. Therefore, we have

$$
\left\{\begin{aligned}
J_{m} \frac{d \omega_{m}}{d t}+b_{m} \omega_{m} & =k_{T} i_{a}-T_{L}, \\
L_{a} \frac{d i_{a}}{d t}+R_{a} i_{a} & =e_{a}-k_{b} \omega_{m}
\end{aligned}\right.
$$

where $k_{T}=k_{p} i_{f}, k_{b}=k_{c} i_{f}$.

When the DC motor is employed to drive the conveyor belt, then,

$$
T_{L}=F_{U} r, \quad V=2 \pi r w_{m},
$$


where $r$ is the radius of the rotor and $F_{u}$ is the peripheral driving force of the blet conveyor and can be calculated by the following equation [18]

$$
\begin{aligned}
& F_{U}=\frac{V T}{3.6}+\frac{T^{2}}{6.48 \rho b_{1}^{2}}+\{g f Q[L \cos \delta \\
& \left.\left.+L(1-\cos \delta)\left(1-\frac{2 Q_{B}}{Q}\right)\right]+k_{3}+C_{F t}\right\} \\
& +k_{1} \frac{T^{2}}{V^{2}}+\left(\frac{g L \sin \delta+g f L \cos \delta}{3.6}+k_{2}\right) \frac{T}{V},
\end{aligned}
$$

where $f$ is the artificial friction factor, $L$ is the centerto-center distance (m), $Q=Q_{R O}+Q_{R U}+2 Q_{B}, Q_{R O}$ is the unit mass of the rotating parts of carrying idler rollers $(\mathrm{kg} / \mathrm{m}), Q_{R U}$ is the unit mass of the belt of rotating parts of the return idler rollers $(\mathrm{kg} / \mathrm{m}), Q_{B}$ is the unit mass of the belt $(\mathrm{kg} / \mathrm{m}), \delta$ is the inclination angle $\left(^{\circ}\right), \rho$ is the bulk density of materia $\left(\mathrm{kg} / \mathrm{m}^{3}\right), b_{1}$ is the width between the skirt boards (m), $k_{1}, k_{2}, k_{3}$ are constants coefficients which relate to the structural parameters of the belt conveyor, $C_{F t}$ is a constant. From (5) and (6), we have

$$
\begin{aligned}
& T_{L}-\frac{2 \pi r^{2} T w_{m}}{3.6}=\frac{r T^{2}}{6.48 \rho b_{1}^{2}}+r\{g f Q[L \cos \delta \\
& \left.\left.+L(1-\cos \delta)\left(1-\frac{2 Q_{B}}{Q}\right)\right]+k_{3}+C_{F t}\right\} \\
& +k_{1} r_{\frac{T^{2}}{4 \pi^{2} r^{2} w_{m}^{2}}}+\left(\frac{g L \sin \delta+g f L \cos \delta}{3.6}+k_{2}\right) \frac{T}{2 \pi r w_{m}} .
\end{aligned}
$$

Let $\quad \theta_{1}=\frac{r}{6.48 \rho b_{1}^{2}}, \quad \theta_{2}=r\{g f Q[L \cos \delta+L(1-$ $\left.\left.\cos \delta)\left(1-\frac{2 Q_{B}}{Q}\right)\right]+k_{3}+C_{F t}\right\}, \quad \theta_{3}=\frac{k_{1} r}{4 \pi^{2} r^{2}}$, $\theta_{4}=\left(\frac{g L \sin \delta}{3.6} \frac{g f L \cos \delta}{3.6}+k_{2}\right) \frac{1}{2 \pi r}, \quad \theta=\left[\theta_{1}, \theta_{2}, \theta_{3}, \theta_{4}\right]^{T}$, and

$$
\psi\left(T, w_{m}\right)=\left[\begin{array}{llll}
T^{2} & 1 & \frac{T^{2}}{w_{m}^{2}} & \frac{T}{w_{m}}
\end{array}\right]^{T} .
$$

Then, a new energy model of belt conveyor with DC motor is given as follows

$$
\left\{\begin{array}{l}
J_{m} \frac{d \omega_{m}}{d t}+b_{m} \omega_{m}=k_{T} i_{a}-\frac{2 \pi r^{2} T w_{m}}{3.6}-\psi^{T}\left(T, w_{m}\right) \theta \\
L_{a} \frac{d i_{a}}{d t}+R_{a} i_{a}=e_{a}-k_{b} \omega_{m}
\end{array}\right.
$$

or

$$
\dot{z}=\tilde{A} z+\tilde{B} u+\tilde{b} \frac{\psi^{T}\left(T, w_{m}\right)}{J_{m}} \theta
$$

where $\tilde{A}=\left[\begin{array}{cc}-\frac{b_{m}}{J_{m}} & \frac{k_{T}}{J_{m}} \\ -\frac{k_{b}}{L_{a}} & -\frac{R_{a}}{L_{a}}\end{array}\right], \quad \tilde{B}=\left[\begin{array}{cc}1 & 0 \\ 0 & 1\end{array}\right], \tilde{b}=$ $\left[\begin{array}{l}1 \\ 0\end{array}\right], z=\left[\begin{array}{c}w_{m} \\ i_{a}\end{array}\right], u=\left[\begin{array}{c}-\frac{2 \pi r^{2}}{3.6 J_{m}} T w_{m} \\ \frac{1}{L_{a}} e_{a}\end{array}\right]$.

For the conveyors with permanent instruments for $T$ and $w_{m}$, the real-time data can be accessed through the supervisory control and data acquisition system (SCADA). Therefore, let $w_{m}$ is the output of the system (8), i.e.,

$$
y=w_{m}=C z,
$$

where $C=\left[\begin{array}{ll}1 & 0\end{array}\right]$. We obtain that

$$
\operatorname{rank}\left[\begin{array}{c}
C \\
C \tilde{A}
\end{array}\right]=\operatorname{rank}\left[\begin{array}{cc}
1 & 0 \\
-\frac{b_{m}}{J_{m}} & \frac{k_{T}}{J_{m}}
\end{array}\right]=2,
$$

which means that $(\tilde{A}, C)$ is observable. Then, the coordinate transformation

$$
\tilde{z}=Q_{1} z=\left[\begin{array}{cc}
1 & 0 \\
-\frac{b_{m}}{J_{m}} & \frac{k_{T}}{J_{m}}
\end{array}\right] z
$$

can transform (9), (10) into the following canonical form [32]

$$
\left\{\begin{array}{l}
\dot{\tilde{z}}_{1}=\tilde{z}_{2}+a_{1} y-\frac{2 \pi r^{2}}{3.6 J_{m}} T w_{m}+\frac{1}{J_{m}} \psi^{T}(T, y) \theta \\
\dot{\tilde{z}}_{2}=a_{2} y-\frac{2 R_{a} \pi r^{2}}{3.6 L_{a} J_{m}} T w_{m}+\frac{k_{T}}{J_{m} L_{a}} e_{a}+\frac{R_{a}}{L_{a} J_{m}} \psi^{T}(T, y) \theta \\
y=\tilde{z}_{1}
\end{array}\right.
$$

where $a_{1}=-\frac{b_{m}}{J_{m}}-\frac{R_{a}}{L_{a}}, a_{2}=-\frac{R_{a} b_{m}}{L_{a} J_{m}}-\frac{k_{T} k_{b}}{J_{m} L_{a}}$. Let $b=$ $\left[1, b_{2}\right]^{T}$ is a vector such that

$$
s+b_{2}
$$

is a Hurwitz polynomial. Consider the following filter transformation [26]

$$
\left\{\begin{array}{l}
x_{1}=\tilde{z}_{1} \\
x_{2}=\tilde{z}_{2}-\sum_{i=1}^{4} \eta_{i} \theta_{i}
\end{array}\right.
$$

where

$$
\dot{\eta}_{i}=-b_{2} \eta_{i}-\frac{b_{2}}{J_{m}} \psi_{i}\left(T, \omega_{m}\right)+\frac{R_{a}}{L_{a} J_{m}} \psi_{i}\left(T, \omega_{m}\right),
$$


where $\eta_{i}\left(t_{0}\right)=0, \psi_{i}\left(T, \omega_{m}\right)$ is the $i$ th component of $\psi\left(T, \omega_{m}\right)(i=1, \cdots, 4)$. It transforms (11) into to the following system

$$
\left\{\begin{aligned}
\dot{x}_{1}= & x_{2}+a_{1} \omega_{m}-\frac{2 \pi r^{2}}{3.6 J_{m}} T w_{m} \\
& +\left(\frac{1}{J_{m}} \psi\left(T, \omega_{m}\right)+\eta\right)^{T} \theta, \\
\dot{x}_{2}= & a_{2} \omega_{m}-\frac{2 R_{a} \pi r^{2}}{3.6 L_{a} J_{m}} T w_{m}+\frac{k_{T}}{J_{m} L_{a}} e_{a} \\
& +b_{2}\left(\frac{1}{J_{m}} \psi\left(T, \omega_{m}\right)+\eta\right)^{T} \theta,
\end{aligned}\right.
$$

where $\eta=\left[\eta_{1}, \eta_{2}, \eta_{3}, \eta_{4}\right]^{T}$. For the system (14) which is in adaptive observer form, an adaptive observer can be designed as follows [26]

$$
\left\{\begin{aligned}
\dot{\hat{x}}_{1}= & \hat{x}_{2}+k_{1} e_{1}+a_{1} \omega_{m}-\frac{2 \pi r^{2}}{3.6 J_{m}} T w_{m} \\
& +\left(\frac{1}{J_{m}} \psi\left(T, \omega_{m}\right)+\eta\right)^{T} \hat{\theta} \\
\dot{\hat{x}}_{2}= & k_{2} e_{1}+a_{2} \omega_{m}-\frac{2 R_{a} \pi r^{2}}{3.6 L_{a} J_{m}} T w_{m} \\
& +\frac{k_{T}}{J_{m} L_{a}} e_{a}+b_{2}\left(\frac{1}{J_{m}} \psi\left(T, \omega_{m}\right)+\eta\right)^{T} \hat{\theta},
\end{aligned}\right.
$$

and

$$
\dot{\hat{\theta}}=\Gamma\left(\frac{1}{J_{m}} \psi\left(T, \omega_{m}\right)+\eta\right) e_{1},
$$

where $e_{1}=x_{1}-\hat{x}_{1}$, and $k_{1}=b_{2}+\lambda, k_{2}=b_{2} \lambda, \lambda>0$, $\Gamma$ is any symmetric positive matrix. From (14) and (15), (16), if follows that

$$
\left\{\begin{array}{l}
\dot{e}_{1}=e_{2}-k_{1} e_{1}+\left(\frac{1}{J_{m}} \psi\left(T, \omega_{m}\right)+\eta\right)^{T} \tilde{\theta} \\
\dot{e}_{2}=-k_{2} e_{1}+b_{2}\left(\frac{1}{J_{m}} \psi\left(T, \omega_{m}\right)+\eta\right)^{T} \tilde{\theta}
\end{array}\right.
$$

and

$$
\dot{\tilde{\theta}}=-\Gamma\left(\frac{1}{J_{m}} \psi\left(T, \omega_{m}\right)+\eta\right) e_{1},
$$

where $e=x-\hat{x}, \tilde{\theta}=\theta-\hat{\theta}$.

Let us now state and prove the main results of this paper.

Theorem 1 For the energy model of belt conveyor with DC motor (8), there exists filter transformation (12), (13) to transform (8) into (14). Moreover, for the system (15), (16), if $k_{1}$ and $k_{2}$ are selected such that $(A, b, C)$ satisfies the strictly positive real condition, where $A=A_{0}+K C, K=\left[k_{1}, k_{2}\right]^{T}$, then, $\|\hat{\theta}(t)-\theta\|$ is uniformly bounded.

Proof: Using the same method as in [26], we can obtain the result.

In order to ensure that $\hat{\theta}(t)$ converges to the desired value, the following result is needed.

Lemma 1 Consider the following system

$$
\left\{\begin{array}{l}
\dot{\eta}=A_{1} \eta+B_{1} \frac{\psi\left(T, \omega_{m}\right)}{J_{m}}, \\
\bar{y}=C_{1} \eta+D_{1} \frac{\psi\left(T, \omega_{m}\right)}{J_{m}},
\end{array}\right.
$$

where $\quad A_{1}=\operatorname{diag}\left\{-b_{2},-b_{2},-b_{2},-b_{2}\right\}, \quad B_{1}=$ $\operatorname{diag}\left\{-b_{2}+\frac{R_{a}}{L_{a}},-b_{2}+\frac{R_{a}}{L_{a}},-b_{2}+\frac{R_{a}}{L_{a}},-b_{2}+\right.$ $\left.\frac{R_{a}}{L_{a}},-b_{2}+\frac{R_{a}}{L_{a}}\right\}, C_{1}=I, D_{1}=I$. If there exist $T_{0}>0$, $k_{p}>0$ such that $\psi\left(T, \omega_{m}\right)$ satisfies the following persistence excitation condition

$$
\int_{t}^{t+T_{0}} \psi\left(T(\tau), \omega_{m}(\tau)\right) \psi^{T}\left(T(\tau), \omega_{m}(\tau)\right) d \tau>k_{p} I,
$$

then, there exists $k_{p}^{\prime}>0$ such that

$$
\int_{t}^{t+T_{0}} \bar{y}(\tau) \bar{y}^{T}(\tau) d \tau>k_{p}^{\prime} I .
$$

Proof: It follows from (19) that

$$
C_{1}\left(S I-A_{1}\right) B_{1}+D_{1}=\left(\frac{s+\frac{R_{a}}{L_{a}}}{s+b_{2}}\right) I,
$$

which implies that the system (19) is stable and minimal phase. By Lemma 2.6.7 in [33], we obtain the result.

Theorem 2 For the energy model of belt conveyor with DC motor (8), there exists filter transformation (12), (13) to transform (8) into (14). Moreover, for the system (15), (16), if $k_{1}$ and $k_{2}$ are selected such that $(A, b, C)$ satisfies the strictly positive real condition, and there exist $T_{0}>0$ and $k_{p}>0$ such that the 
condition (20) holds, then, we have $\lim _{t \rightarrow \infty}\|\theta-\hat{\theta}(t)\|=$ 0.

Proof: From (13), we have

$\eta_{i}(t)=e^{-b_{2} t} \int_{t_{0}}^{t} e^{b_{2} \tau}\left(-\frac{b_{2}}{J_{m}}+\frac{R_{a}}{L_{a} J_{m}}\right) \psi_{i}\left(T(\tau), \omega_{m}(\tau)\right) d \tau$.

It is obvious that $\psi\left(T, \omega_{m}\right)$ is bounded for every $\left(T, w_{m}\right)$ bounded. Therefore, $\eta_{i}(t)(i=1,2,3,4)$ are bounded for every $\left(T, w_{m}\right)$, which implies that $\left(\frac{1}{J_{m}} \psi\left(T, \omega_{m}\right)+\eta\right)$ is bounded for every $\left(T, w_{m}\right)$ bounded. Along the trajectory of the system (17), (18), calculate the derivative of the following Lyapunov function

$$
V(e)=e^{T} P e+\tilde{\theta}^{T} \Gamma^{-1} \tilde{\theta},
$$

we have

$$
\begin{gathered}
\left.\frac{d V(e)}{d t}\right|_{(17),(18)}=e^{T}\left(A^{T} P+P A\right) e \\
+2 e^{T} P b\left(\frac{1}{J_{m}} \psi(T, y)+\eta\right)^{T} \tilde{\theta} \\
-\tilde{\theta}^{T}\left(\frac{1}{J_{m}} \psi(T, y)+\eta\right) e_{1}<-d e^{T} Q e .
\end{gathered}
$$

Thus, $\|e(t)\|$ and $\|\tilde{\theta}(t)\|$ are uniformly bounded for any $t>t_{0}$. Moreover, $\left(\frac{1}{J_{m}} \psi(T, y)+\eta\right)$ is uniformly bounded, then, $\|\dot{e}(t)\|$ is also uniformly bounded. Since $V(e)$ is a uniformly bounded non-increasing function

$$
\lim _{t \rightarrow \infty} \int_{t_{0}}^{t} e^{T}(\tau) Q e(\tau) d \tau<V\left(t_{0}\right)-V(\infty)<V(\infty),
$$

By Barbalat Lemma [26], we have

$$
\lim _{t \rightarrow \infty}\|e(t)\|=0
$$

From (18) and (23), we have

$$
\lim _{t \rightarrow \infty} \dot{\tilde{\theta}}(t)=0
$$

Then, there exits a constant $\tilde{\theta}^{*}$ such that

$$
\lim _{t \rightarrow \infty} \tilde{\theta}(t)=\tilde{\theta}^{*}
$$

Therefore, for any $\varepsilon>0$, there exists $t_{1}>0$ such that

$$
\left\|\tilde{\theta}(t)-\tilde{\theta}^{*}\right\|<\varepsilon, \forall t>t_{1} .
$$

Now, we will prove that $\tilde{\theta}^{*}=0$ by contradiction. Assume that $\tilde{\theta}^{*} \neq 0$.

Consider the following function

$\varphi(\tilde{\theta}(t), t)=\frac{1}{2}\left[\tilde{\theta}^{T}\left(t+T_{0}\right) \Gamma^{-1} \tilde{\theta}\left(t+T_{0}\right)-\tilde{\theta}^{T}(t) \Gamma^{-1} \tilde{\theta}(t)\right]$,

which is bounded. The time derivative of $\varphi(\tilde{\theta}(t), t)$ is given as

$$
\begin{aligned}
\frac{d \varphi(\tilde{\theta}(t), t)}{d t}= & \tilde{\theta}^{T}\left(t+T_{0}\right) \Gamma^{-1} \dot{\tilde{\theta}}\left(t+T_{0}\right)-\tilde{\theta}^{T}(t) \Gamma^{-1} \dot{\tilde{\theta}}(t) \\
& =\int_{t}^{t+T_{0}} \frac{d}{d \tau}\left(\tilde{\theta}^{T}(\tau) \Gamma^{-1} \dot{\tilde{\theta}}(\tau)\right) d \tau \\
= & -\int_{t}^{t+T_{0}} \frac{d}{d \tau}\left(\tilde{\theta}^{T}(\tau) \bar{y}(\tau) e_{1}(\tau)\right) d \tau \\
= & \int_{t}^{t+T_{0}} e_{1}(\tau) \bar{y}^{T}(\tau) \Gamma \bar{y}(\tau) e_{1}(\tau) d \tau \\
& -\int_{t}^{t+T_{0}} \tilde{\theta}^{T}(\tau) \dot{\bar{y}}(\tau) e_{1}(\tau) d \tau \\
- & \int_{t}^{t+T_{0}} \tilde{\theta}^{T}(\tau) \bar{y}(\tau)\left(e_{2}(\tau)-k_{1} e_{1}(\tau)\right) d \tau \\
& \left.-\int_{t}^{t+T_{0}} \tilde{\theta}^{T}(\tau) \bar{y}(\tau) \bar{y}^{T}(\tau)\right) \tilde{\theta}(\tau) d \tau .
\end{aligned}
$$

Note that $\dot{\bar{y}}(\tau)=\left(\frac{1}{J_{m}} \dot{\psi}(T(\tau), y(\tau))+\dot{\eta}(\tau)\right)$ is uniformly bounded for every $\left(T, w_{m}\right)$ bounded, and $\lim _{t \rightarrow \infty} e(t)=0$, and (21), (24) hold, then, there exists $M>0$ such that when $t>t_{1}$, we have

$$
\begin{gathered}
\frac{d \varphi(\tilde{\theta}(t), t)}{d t}<M \int_{t}^{t+T_{0}}\left(e_{1}^{2}(\tau)+e_{2}(\tau)^{2}\right) d \tau \\
-\int_{t}^{t+T_{0}} \tilde{\theta}^{* T} \bar{y}(\tau) \bar{y}^{T}(\tau) \tilde{\theta}^{*} d \tau \\
-2 \int_{t}^{t+T_{0}} \tilde{\theta}^{* T} \bar{y}(\tau) \bar{y}^{T}(\tau)\left(\tilde{\theta}(\tau)-\tilde{\theta}^{*}\right) d \tau \\
-\int_{t}^{t+T_{0}}\left(\tilde{\theta}(\tau)-\tilde{\theta}^{*}\right)^{T} \bar{y}(\tau) \bar{y}^{T}(\tau)\left(\tilde{\theta}(\tau)-\tilde{\theta}^{*}\right) d \tau \\
<M \int_{t}^{t+T}\left(e_{1}^{2}(\tau)+e_{2}(\tau)^{2}\right) d \tau \\
-\frac{k_{p}^{\prime}}{2} \tilde{\theta}^{* T} \tilde{\theta}^{*}<-\frac{k_{p}^{\prime}}{4} \tilde{\theta}^{* T} \tilde{\theta}^{*}, \forall t>t_{1},
\end{gathered}
$$

which contradicts the bounbedness of $\varphi(\tilde{\theta}(t), t)$. Therefore, $\lim _{t \rightarrow \infty} \tilde{\theta}(t)=0$. The proof is completed. 


\section{Simulation Results}

We test the proposed adaptive parameter estimation (13), (15), (16) by simulation with parameters $\quad b_{2}=4.0, \quad \lambda=3, \quad \Gamma=$ $\operatorname{diag}\{180,180,180,180\}$ and with four coefficients $\theta_{1}=1, \theta_{2}=0.3, \theta_{3}=3.5, \theta_{4}=2.1$, for a DC motor, whose parameters are: $k_{T}=0.1, k_{b}=0.1, b_{m}=0.4$, $J_{m}=0.05 \quad \mathrm{Kgm}^{2}, \quad R_{a}=15 \quad \mathrm{Ohm}, \quad L_{a}=1.0 \mathrm{H}$, $r=0.01 \mathrm{~m}, e_{a}=380 \mathrm{~V}$. The initial condition of (14), (15), and (16) are given by $(0.01,0.7),(0.8,0.2)$ and $(0.1,0.1,0.1,0.1)$, respectively. It should be noted that it is difficult to check the inequality (20) holds. In practice, if the feed rate $T$ does not change much, a complete determination of all the parameters is impossible. In order to estimate all the parameters, one should sufficiently disturbing the feed rate $T$ during the period of estimation. In this example, we choose the feed rate $T=0.09(8+5 \sin (10 t+1)+2 \cos (-5 t+$ $2)+\sin (20 t))+0.3(8.6+2 \cos (-5 t+2)+\sin (15 t+$ $0.4)+\sin (20 t)+4 \sin (t))|(\sin (4 t+0.5))| \quad \mathrm{kg} / \mathrm{s}$ $(0 \leq t \leq 60 s)$. The simulation results are shown in Fig. 3-Fig. 6.

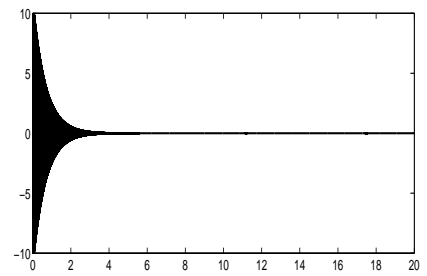

Fig. 3. Trajectory of $\tilde{\theta}_{1}$.

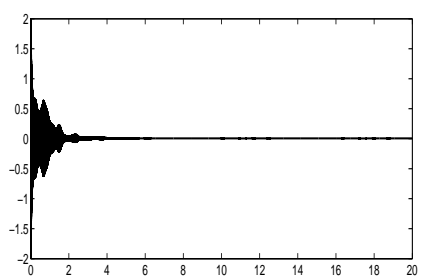

Fig. 4. Trajectory of $\tilde{\theta}_{2}$.

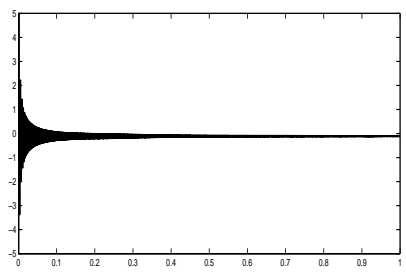

Fig. 5. Trajectory of $\tilde{\theta}_{3}$.

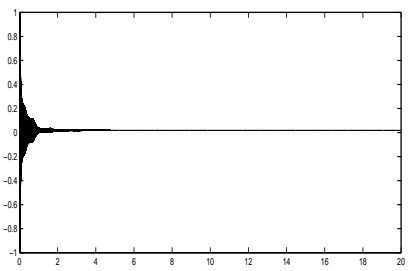

Fig. 6. Trajectory of $\tilde{\theta}_{4}$.

To test the algorithm against measurement noise, a band limited white noise is added to $y_{1}$. The results are demonstrated in Fig. 7-Fig. 10.

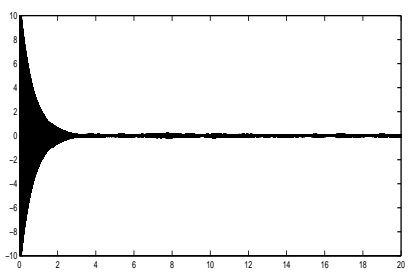

Fig. 7. Trajectory of $\tilde{\theta}_{1}$ with band limited white noise.

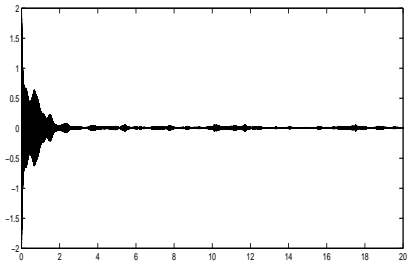

Fig. 8. Trajectory of $\tilde{\theta}_{2}$ with band limited white noise.

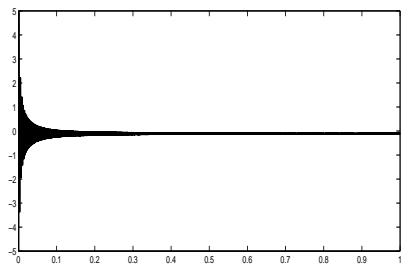

Fig. 9. Trajectory of $\tilde{\theta}_{3}$ with band limited white noise. 


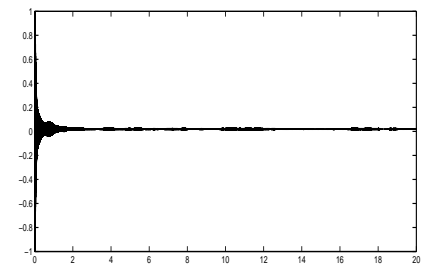

Fig. 10. Trajectory of $\tilde{\theta}_{4}$ with band limited white noise.

Practically, the parameters may drift away during the belt conveyor operates. For example, $\theta_{1}=1.2, \theta_{2}=$ $0.3, \theta_{3}=3.5, \theta_{4}=2.3$, using the same initial conditions and the feed rate $T$, we implement the adaptive identifer for 30s. Fig. 11- Fig. 14 show the simulation results.

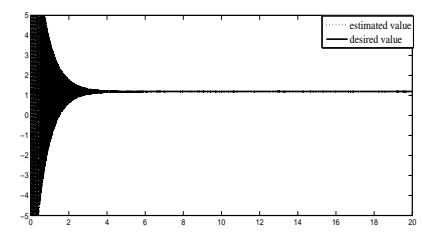

Fig. 11. Trajectories of $\theta_{1}$ and $\hat{\theta}_{1}$.

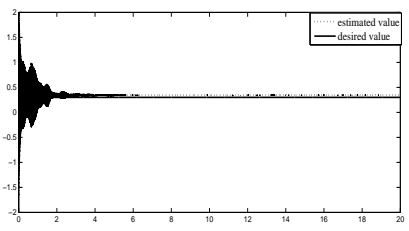

Fig. 12. Trajectories of $\theta_{2}$ and $\hat{\theta}_{2}$.

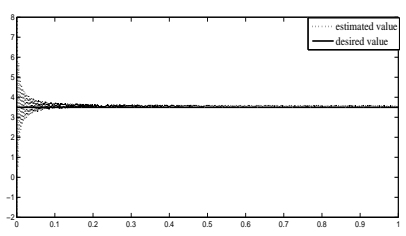

Fig. 13. Trajectories of $\theta_{3}$ and $\hat{\theta}_{3}$.

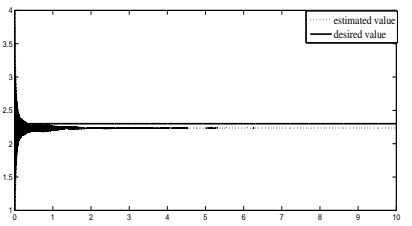

Fig. 14. Trajectories of $\theta_{4}$ and $\hat{\theta}_{4}$.

\section{Conclusion}

In this paper, a new energy model of a belt conveyor driven by a DC motor was presented, which lumped all the parameters into four coefficients. Then, an adaptive observer was designed to estimate the unknown parameters. In addition, under a persistent excitation condition, the convergence of the parameters to the desired values could also be concluded. Compared with the existing methods, our methods could be implemented by measuring only the feed rate of the belt conveyor and the angular velocity of the rotor of the DC motor.

\section{REFERENCES}

1. Marais J, Mathews E, Pelzer R, "Analysing DSM opportunities on mine conveyor systems," In: Industrial and Commercial Use of Energy Conference, Cape Town, South Africa (2008).

2. Hager M, Hintz A, "The energy saving design of belts for long conveyor systems," Bulk Solids Handl, vol. 13, no. 4, pp. 749-758 (1993).

3. Xia X, Zhang J, "Control systems and energy efficiency from the POET perspective," In: IFAC Conference on Control Methodologies and Technology for Energy Efficiency; Vilamoura, Portugal; pp. 29-31 (2010).

4. Reicks AV, "Belt conveyor idler roll behavours," In: Alspaugh MA, editor. Bulk material handling by conveyor belt 7. Colorado, pp. 35-40 (2008).

5. Staniak K, França, Jr J, "Energy saving in long distance conveyors-Novel idler technology," In: Hennies WT, Ayres da Silva LA, Chaves AP, editors. Mine planning and equipment selection. 
Rotterdam, Netherlands: Balkema, pp. 479-486 (1996).

6. Tapp AG, "Energy saving troughing idler technology," Bulk Solids Handl, vol. 20, no. 4, pp. 437-449 (2000).

7. Jansen M, "The development of energy-optimized conveyor belts-a joint project of the conveyor belt group of ContiTech AG and RWE power AG," World Min, vol. 60, no. 2, pp. 83-87 (2008).

8. De Almeida AT, Fonseca P, Bertoldi P, "Energyefficient motor systems in the industrial and in the services sectors in the European Union: characterisation, potentials, barriers and policies," Energy, vol. 28, pp. 673-90 (2003).

9. Middelberg A, Zhang J, Xia X, “An optimal control model for load shifting - with application in the energy management of a colliery," Appl Energy, vol. 86, pp. 1266-1273, (2009).

10. Marx DJL, Calmeyer JE, "An integrated conveyor energy model methodology," Trans South African Inst Electr Eng, vol. 95, pp. 256-264, (2004).

11. Marx DJL, Calmeyer JE, "A case study of an integrated conveyor belt model for mining industry," In: IEEE AFRICON 2004; Gaborone, Botswana, pp. 15-17 (2004).

12. Lauhoff H, "Speed control on belt conveyors-dose is really save engery?" Bulk Solids Handl, vol. 26, no. 6, pp. 68-77 (2005).

13. Van Den Heuvel B, "Investigations dealing with variable-speed drives of belt conveyor systems," World Min, vol. 58, no. 2, pp. 105-113 (2006).

14. Daubs W, Köerber S, Becher N, "Raw coal loading and belt conveyor system at Nochten opencast mine - a new conveying and loading system based on drives controlled and adjusted by frequency converter," Braunkohle Surf Min, vol. 50, no. 2, pp. 117-130 (1998).

15. Köhler U, Sykulla M, Wuschek V, "Variable-speed belt conveyors gaining in importance," Braunkohle Surf Min, vol. 53, no. 1, pp. 65-72 (2001).

16. Koopmann K, Weber D, Keller C, Daus W, Köber $\mathrm{S}$, "Conveying device for opencast mines," US Patent, 6209711 B1 (2001).

17. Zhang S, Xia X, "Optimal control of operation efficiency of belt conveyor systems," Appl Energy, vol. 87, no. 6, pp. 1929-1937 (2010).

18. Zhang S, Xia X, "Modeling and energy efficiency optimization of belt conveyors," Appl Energy, vol. 88, no. 9, pp. 3061-3071 (2011).

19. Ljung L, System Identification Theory For The User. 2nd ed. New Jersey: Prentice-Hall Inc. (1999).

20. Koubaa Y, "Recursive identification of induction motor parameters," Simul Modell Pract Theory, vol. 12, pp. 363-381, (2004).

21. Lüders G, Narendra K S, "An adaptive observer and identifier for a linear system," IEEE Trans. Automat. Contr., vol. 18, pp. 496-499 (1973).

22. Kreisselmeier G, "Adaptive observers with exponential rate of convergence," IEEE Trans. Automat. Contr., vol. 22, pp. 2-8 (1977).

23. Narendra K S, Annaswamy A, Stable Adaptive Systems, New Jersey: Prentice-Hall Inc. (1989).

24. Zhang Q, "Adaptive observers for mimo linear time-varying systems," IEEE Trans. Automat. Contr., vol. 47, pp. 1202-1204 (2002).

25. Bastin G, Gevers M, "Stable adaptive observers for nonlinear time varying systems," IEEE Trans. Automat. Contr., vol. 33, pp. 650-658 (1988).

26. Marino R, Tomei P, Nonlinear Control Design: Geometric, Adaptive and Robust. New Jersey: 
Prentice-Hall Inc. (1995).

27. Marino R., Tomei P, "Adaptive observers with arbitrary exponential rate of convergence for nonlinear systems," IEEE Trans. Automat. Contr., vol. 40, pp. 1300-1304 (1995).

28. Xu A, Zhang Q, "Nonlinear system fault diagnosis based on adaptive estimation," Automatica, vol. 40, 1183-1193 (2004).

29. Zhang X, Yan L, "An adaptive output feedback dynamic surface control for a class of nonlinear systems with unknown backlash-like hysteresis," Asial Journal of Control, vol. 16, 1-12 (2012).

30. Sang $Q$ and Tao G, "Adaptive Control of Piecewise Linear Systems with State Feedback for Output Tracking," Asian Journal of Control. doi: 10.1002/asjc.596. (2012)

31. Stadler W, Analytical Robotics and Mechatronics. New York: McGraw Hill (1995).

32. Tsay Y, Shieh L, "Block decompositions and block modal controls, of multivariable control systems," Automatica, vol. 19, pp. 29-40 (1983).

33. Sastry S, Bodson M, Adaptive Control, Stability, Convergence, Robustness, New Jersey: PrenticeHall Inc. (1989). 